\title{
RESEARCH
}

Open Access

\section{Predictors of responders to mononuclear stem cell-based therapeutic angiogenesis for no-option critical limb ischemia}

Tianyue Pan ${ }^{1,2 \dagger}$, Hao Liu, ${ }^{1,2 \dagger}$, Yuan Fang ${ }^{1,2}$, Zheng Wei ${ }^{3}$, Shiyang Gu ${ }^{3}$, Gang Fang ${ }^{1,2}$, Yifan Liu, L $^{1,2}$, Yang Luo ${ }^{1,2}$,

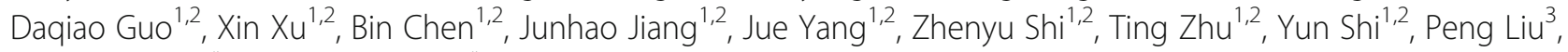
Zhihui Dong ${ }^{1,2^{*}}$ and Weiguo $\mathrm{Fu}^{1,2^{*}}$

\begin{abstract}
Background: Although the mononuclear cell (MNC) transplantation could theoretically induce therapeutic angiogenesis in the patients with no-option critical limb ischemia (NO-CLI), the clinical responses to this approach are inconsistent among different clinical trials. The purpose of this study was to identify the prognostic factors of responders and develop a predictive nomogram to guide patient selection.
\end{abstract}

Methods: We retrospectively reviewed a consecutive NO-CLI cohort who received peripheral blood-derived transplantation in our center. The patients who survived and achieved complete remission of CLI at 6 months post-transplantation were defined as responders. Logistic regression models were used to screen and identify the prognostic factors based on which predictive nomogram was developed. A receiver operating characteristic (ROC) curve and a calibration curve were drawn to determine the discrimination level and predictive accuracy.

Results: The study ultimately enrolled 103 patients, including 58 responders and 45 non-responders. Based on the multivariate regression analysis, age $\geq 50$ years (odds ratio [OR] 0.201, $P=0.004$ ), blood fibrinogen $>4 \mathrm{~g} / \mathrm{L}$ (OR 0.176 , $P=0.003)$, arterial occlusion above the knee/elbow (OR 0.232, $P=0.010)$, the transcutaneous pressure of oxygen $\left(\mathrm{TCPO}_{2}\right)(\mathrm{OR} 1.062, P=0.006)$, and the Log total transplanted $\mathrm{CD} 34^{+}$cell count $(\mathrm{OR} 3.506, P=0.046)$ were identified as independent prognostic factors of the responders in the nomogram. An area under the ROC curve of 0.851 indicated good discrimination, and the calibration curve of the predicted probability showed optimal agreement with that of the observed probability.

Conclusions: Age, blood fibrinogen, arterial occlusion level, $\mathrm{TCPO}_{2}$, and the total transplanted $\mathrm{CD} 34^{+}$cell count were independent prognostic factors of the responders. A nomogram with high discrimination and accuracy was developed to provide individualized predictions.

Trail registration: ChiCTR, ChiCTR1800019401. Registered 9 November 2018-Retrospectively registered Keywords: Critical limb ischemia, Therapeutic angiogenesis, Mononuclear cell transplantation, Predictive analysis, Nomogram

\footnotetext{
* Correspondence: dzh926@126.com; fu.weiguo@zs-hospital.sh.cn

†Tianyue Pan and Hao Liu contributed equally to this work.

${ }^{1}$ Department of Vascular Surgery, Zhongshan Hospital, Fudan University,

Shanghai 200032, China

Full list of author information is available at the end of the article
}

(c) The Author(s). 2019 Open Access This article is distributed under the terms of the Creative Commons Attribution 4.0 International License (http://creativecommons.org/licenses/by/4.0/), which permits unrestricted use, distribution, and reproduction in any medium, provided you give appropriate credit to the original author(s) and the source, provide a link to the Creative Commons license, and indicate if changes were made. The Creative Commons Public Domain Dedication waiver (http://creativecommons.org/publicdomain/zero/1.0/) applies to the data made available in this article, unless otherwise stated. 


\section{Background}

As the terminal stage of peripheral artery disease (PAD), critical limb ischemia (CLI), which is characterized by rest pain and unhealing wounds of the limbs, is associated with a 1-year major amputation rate of $30 \%$ and mortality rate of $25 \%[1,2]$. Currently, endovascular and surgical revascularizations are the effective mainstream interventional strategies for CLI [3]. However, up to $30 \%$ of CLI patients are not suitable candidates for these procedures due to distal runoff deficiency or rapid postoperative arterial re-occlusion [4]. In these so-called no-option CLI (NO-CLI) patients, the prognosis appears to be even worse, with a major amputation rate of $40 \%$ at 6 months from onset [2]. Novel approaches of stem cell-based therapeutic angiogenesis, including bone marrow- (BM) or peripheral blood (PB)-derived mononuclear cell (MNC) transplantation with or without purification of specific cell types, are increasingly being used in clinical trials that attempt to treat NO-CLI [513]. These phase I/II trials have confirmed the safety and feasibility of MNC transplantation and revealed its potential therapeutic benefits. However, the curative effects are inconsistent among different study populations. Several clinical trials have shown the positive therapeutic efficacy of $\mathrm{MNC}$ or purified $\mathrm{CD} 34^{+}$cell transplantation in treating NO-CLI patients with respect to avoiding major amputations and promoting wound healing $[12,14,15]$. In contrast, other trials have observed an insignificant moderate prognosis following such therapeutic approaches relative to conservative treatments or placebo $[7,11]$. These controversial results might be attributed largely to the considerable heterogeneity of the study population. Younger patients with thromboangiitis obliterans (TAO) have been shown to achieve more therapeutic benefits than older patients with arteriosclerosis obliterans (ASO) [11, 16]. Notably, aging was considered to be a critical factor for attenuating blood perfusion restoration after cell transplantation [17]. In addition, the concomitant cardiovascular diseases and organ dysfunction would probably lower the survival rate of the elderly PAD/CLI population, thereby compromising the therapeutic benefits of the MNC treatment. Another important factor relating to prognosis is the feature of the transplant. Although the therapeutic mechanism of mononuclear cells remains to be clarified, the dosage of transplanted $\mathrm{CD}^{+} 4^{+}$cells is regarded as a key factor for ineffective revascularization and blood supply restoration [18]. Meta-analyses have revealed that in clinical trials, patients do not respond favorably to MNC transplantation with a relatively low dosage of CD34 ${ }^{+}$cells or MNCs $[19,20]$.

To date, the characteristics of the patients who benefit from MNC transplantation have not been comprehensively investigated. Due to the conflicting findings of different clinical trials, the patient selection criterion of this approach should be reexamined and clarified. This study was performed to explore the clinical background features and transplants of both responders and non-responders to $\mathrm{MNC}$ transplantation and to develop a nomogram to practically formulate an individualized prognostic prediction and clarify the criterion.

\section{Methods \\ Patients}

A consecutive cohort from May 2009 to October 2017 was retrospectively enrolled in our center and included 110 patients with NO-CLI who received peripheral blood mononuclear cell (PBMNC) transplantation with or without purification for $\mathrm{CD} 34^{+}$cells. The patients included in the study met the following inclusion criteria: (1) provision of signed informed consent, (2) male or female aged between 18 and 80 years, (3) diagnosis of limb ischemia due to arterial stenosis or occlusion, as confirmed by computed tomographic angiography, magnetic resonance angiography, or digital subtraction angiography, (4) rest pain or tissue loss under the superior level of metatarsus (Rutherford class 4 or 5) that was anatomically unsuitable for surgery or an endovascular intervention or showing no improvement for at least 3 months following surgery or an endovascular intervention, (5) if present, unrelieved rest pain or a wound size that was not reduced after at least 1 month of medical treatment or other conservative treatment including smoking cessation, dietary control, and exercise therapy, and (6) complete baseline data and known status of survival, amputation, wound healing, and rest pain relief at 6 months after transplantation. The exclusion criteria were (1) serious health events $<3$ months before admission, including but not limited to a myocardial infarction, cerebral apoplexy, a pulmonary embolism, and severe hepatic and renal dysfunction, (2) a diagnosis or suspicion of malignancy at baseline, or (3) a life expectancy $<6$ months.

This study was approved by the ethics committee of Zhongshan Hospital, Fudan University, China. It was performed in agreement with the ethical principles of the Declaration of Helsinki.

\section{Procedures for cell transplantation}

All the patients received subcutaneous injections of rhG-CSF (Neupogen ; Amgen, Thousand Oaks, CA, USA) $(5-10 \mu \mathrm{g} / \mathrm{kg}$ per day for 4 days $)$ to mobilize the BM cells, and enoxaparin (4000 IU/day) was administered daily to prevent hypercoagulable states. On the fifth day, a suspension of PBMNCs $(200 \mathrm{~mL})$ was collected via leukapheresis (COM.TEC; Fresenius Hemocare $\mathrm{GmbH}$, Bad Homburg, Germany). The non-purified PBMNC transplants were obtained by washing three 
times and resuspending the apheresis products in an ethylenediaminetetra-acetic acid-phosphate buffered saline solution $(200 \mathrm{~mL})$ that contained $0.5 \%$ human albumin. The purified $\mathrm{CD} 34^{+}$cell transplants $(40 \mathrm{ml})$ were obtained using a magnetic cell sorting system (MiltenyiBiotec $\mathrm{GmbH}$, BergischGladbach, Germany) immediately after resuspension. The $\mathrm{CD}_{3} 4^{+}$cell counts of all the transplants were determined by leukocyte counting and flow cytometry using CD34 antibody. The final implanted CD34+ cells was set at a dosage ranging from $10^{5}$ to $10^{6}$ per kilogram body weight and the cells were transplanted into the ischemic limbs via equidistant intramuscular injections $(0.5 \mathrm{~mL} / \mathrm{site})$ under general anesthesia. The injections were distributed in the calves/forearms and feet/hands in the patients with arterial occlusions above the ankle/wrist. In those with occlusions below the ankle/wrist, the injections were only administered in the feet/hands.

\section{Baseline information and endpoints}

The baseline information was collected from the medical record of each patient, and this information included the demographic data, the etiology of the limb ischemia, the risk factors for peripheral artery and cardiovascular diseases, the critical results of blood examination, and the treatment history. The baseline features of the treated limb were also recorded, including the Rutherford class, the wound type, the ankle-brachial index (ABI), the transcutaneous pressure of oxygen $\left(\mathrm{TcPO}_{2}\right)$ of the dorsum, and the occlusion level of the arteries according to the pretherapy radiological imaging. The primary endpoint was CLI remission, defined as surviving patients with complete wound healing and rest pain relief, without major limb amputation at 6 months after transplantation. The patients who achieved CLI remission were regarded as responders. The other patients were regarded as non-responders. The secondary endpoints included mortality and the major amputation-free survival rate at 6 months.

\section{Statistical analysis}

The baseline characteristics of the responders and non-responders were compared first. Continuous variables were presented as the means \pm standard deviations (SDs) or as the medians \pm interquartile ranges (IQRs) according to the data distribution. The independent Student's $t$ test or Mann-Whitney $U$ test was used to analyze the significance of the differences between responders and non-responders. A paired $t$-test or Wilcoxon signed-rank test was used to analyze the longitudinal changes from baseline to 6 months post-transplantation. Categorical variables were presented as numbers with percentages, and the Pearson chi-square test or Fisher's exact test was used to analyze the significance of the differences. Subsequently, the candidate prognostic factors of the responders were screened through a univariate binary logistic regression analysis. The correlations between the candidate predictors or the variables at different time points were analyzed with linear regression or Pearson's chi-square test. Furthermore, a multivariate binary logistic regression model was used to identify the independent predictors. Accordingly, a nomogram was developed, followed by performance of the receiver operating characteristic (ROC) curve with the area under the curve being used to evaluate the discrimination level. The calibration curve, which was adjusted with 1000 cycles of bootstrap resampling, was used to analyze the agreement between the predicted and the observed probability of the responders. A two-tailed $P$ value $<0.05$ was considered statistically significant. The statistical analyses were performed with SPSS version 19.0 for Windows (SPSS, Chicago, IL, USA), and the nomogram and the calibration curve were drawn using $\mathrm{R}$ software (version 3.4.3) with rms package (http://www.r-project.org).

\section{Results}

\section{Baseline information and endpoints}

Of the 110 patients reviewed, 3 were lost to follow-up within 6 months, and the critical baseline information of the other 4 was lacking. Therefore, 103 patients with complete data at the 6-month follow-up were finally included and analyzed (Fig. 1). The mean age of the patients was $44.9 \pm 13.1$ years, and the male ratio was $98.1 \%$. The etiologies included TAO (86 patients), ASO (10 patients), collagen disease (4 patients), Crohn's disease (1 patient), and eosinophilia ( 2 patients) (Table 1$)$. There were 99 lower limbs and 4 upper limbs involved in the study. The baseline Rutherford class was 5 in 91 patients $(88.4 \%)$ and 4 in 12 patients $(11.6 \%)$ (Table 1 ). The median total $\mathrm{CD} 34^{+}$cell count in the transplants was $43.0 \times 10^{6}$, with an IQR of $26.0-84.0 \times 10^{6}$. The 6 -month major amputation-free survival rate was $92.2 \%$ (95/103), wherein 3 patients with TAO and 4 patients with ASO underwent major amputations (6 above-the-knee cases and 1 below-the-knee case) and 1 patient with TAO died at 2 months due to hepatic failure of unknown cause. A total of 56.3\% (58/103) patients achieved complete remission from CLI at 6 months and were regarded as responders to the MNC transplantation (Table 1).

\section{Characteristics of responders and non-responders}

The mean age of the responders tended to be lower than that of the non-responders, though the difference was not significant $(43.4 \pm 11.2$ versus $46.8 \pm 15.2$ years, $P=$ 0.204). However, the frequency of the patients whose ages were $<50$ years in the responder group was significantly higher than that in the non-responder group (77.6\% versus $48.9 \%, P=0.002$ ) (Table 1 ). Similarly, 


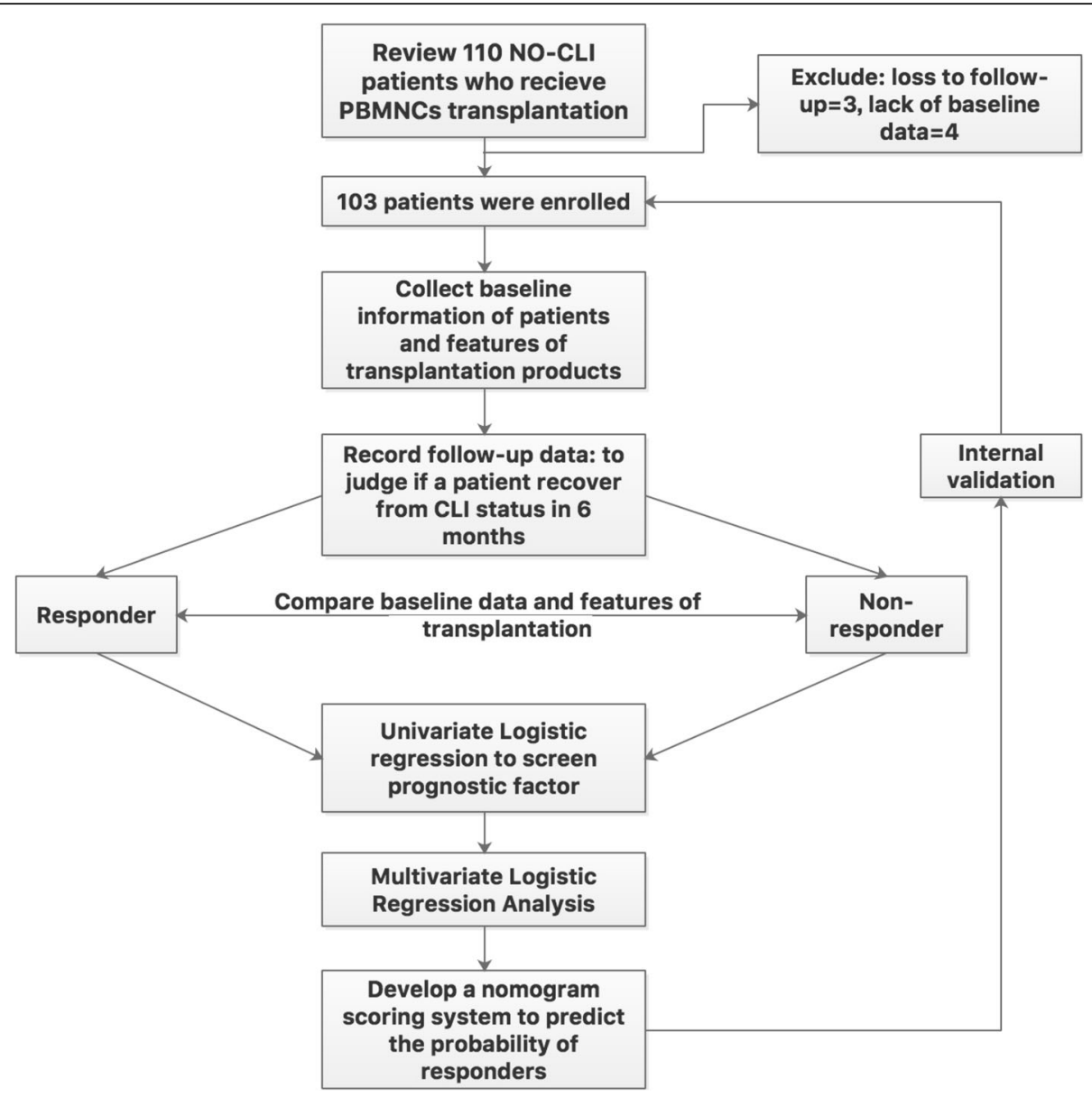

Fig. 1 Study flow diagram. NO-CLI, no-option critical limb ischemia

comparing frequency distribution trends of age in the responders and non-responders, an intersection point was observed at 50 (Additional file 1). When the baseline blood examinations were evaluated, the responders had significantly lower blood fibrinogen and CRP levels than the non-responders (Table 1). No significant differences were observed between the responders and non-responders in terms of the etiologies, risk factors of cardiovascular disease, or treatment histories (Table 1).

Regarding the treated limbs, the responders were characterized by significantly higher baseline ABI (median 0.515, IQR 0.410-0.695) and $\mathrm{TcPO}_{2}$ (median $24 \mathrm{mmHg}$, IQR $14-30 \mathrm{mmHg}$ ) than the non-responders (ABI, median 0.430, IQR 0.280-0.540, $P=0.011 ; \mathrm{TcPO}_{2}$, median $9 \mathrm{mmHg}$, IQR 4-19 mmHg, $P<0.001$ ) (Table 2). Based on the available complete longitudinal data $(n=69)$, the median $\mathrm{TcPO}_{2}$ of the responders increased from $24 \mathrm{mmHg}$ (IQR 14-31 mmHg) at baseline to $42 \mathrm{mmHg}$ (IQR 21-57 $\mathrm{mmHg})$ at 6 months post-transplantation $(P<0.001)$, while that of the non-responders did not improve significantly, increasing from $11.5 \mathrm{mmHg}$ (IQR $4-26 \mathrm{mmHg}$ ) at baseline to $13.5 \mathrm{mmHg}$ (IQR $5-43 \mathrm{mmHg}$ ) at 6 months post-transplantation $(P=0.094) \quad$ (Fig. 2a). $\mathrm{TcPO}_{2}$ at baseline correlated significantly with that at 6 months $\left(R^{2}=0.245, P<0.001\right)$ (Fig. $\left.2 \mathrm{~b}\right)$. Considering the aspect of arterial lesions, $60.02 \%$ of the patients were characterized by an occlusion level above the knee or elbow, including $3.9 \%$ in the common or external iliac artery, $4.8 \%$ in the common femoral artery or axillary artery, $35.0 \%$ in the superficial femoral artery or brachial artery, and $16.5 \%$ in the popliteal artery. The remaining $39.8 \%$ of the patients were characterized by a highest occlusion level below the knee, with $27.2 \%$ of occlusions located in the calves or forearms and $12.6 \%$ of occlusions located below the ankles or wrists. The responders had a significantly lower frequency of arterial occlusions above the knee or elbow than the non-responders (46.6\% versus $77.8 \%, P=0.001$ ) (Table 2 ). In addition, no differences were observed between the responders and non-responders in terms of the Rutherford class $(P=$ $0.277)$ or gangrene $(P=0.718)$ (Table 2$)$. Regarding the transplants, the responders were not associated with preconditioning of $\mathrm{CD} 34^{+}$cell purification $(P=$ 
Table 1 Baseline characteristics of patients

\begin{tabular}{|c|c|c|c|c|}
\hline & Total, $n=103$ & Responders, $n=58$ & Non-responders, $n=45$ & $P$ value \\
\hline Age (years) (mean $\pm S D$ ) & $44.9 \pm 13.1$ & $43.4 \pm 11.2$ & $46.8 \pm 15.2$ & 0.204 \\
\hline $20-29, n(\%)$ & $16(15.5 \%)$ & $8(13.8 \%)$ & $8(17.8 \%)$ & 0.596 \\
\hline $30-39, n(\%)$ & $18(17.5 \%)$ & $13(22.4 \%)$ & $5(11.1 \%)$ & 0.191 \\
\hline $40-49, n(\%)$ & $33(32.0 \%)$ & $24(41.4 \%)$ & $9(20.0 \%)$ & 0.033 \\
\hline $50-59, n(\%)$ & $25(24.3 \%)$ & $10(17.2 \%)$ & $15(33.3 \%)$ & 0.068 \\
\hline $60-69, n(\%)$ & $4(3.9 \%)$ & $0(0.0 \%)$ & $4(8.9 \%)$ & 0.034 \\
\hline 70-79, n (\%) & $7(6.8 \%)$ & $3(5.2 \%)$ & $4(8.9 \%)$ & 0.696 \\
\hline$<50, n(\%)$ & $67(65.0 \%)$ & $45(77.6 \%)$ & $22(48.9 \%)$ & 0.002 \\
\hline$\geq 50, n(\%)$ & $36(35.0 \%)$ & $13(22.4 \%)$ & $23(51.1 \%)$ & 0.002 \\
\hline \multicolumn{5}{|c|}{ Gender } \\
\hline Male, $n(\%)$ & $101(98.1 \%)$ & $56(96.6 \%)$ & $45(100.0 \%)$ & 0.208 \\
\hline Female, $n(\%)$ & $2(1.9 \%)$ & $2(3.4 \%)$ & $0(0.0 \%)$ & 0.208 \\
\hline Body mass index $\left(\mathrm{kg} / \mathrm{m}^{2}\right)($ mean $\pm \mathrm{SD})$ & $23.3 \pm 3.1$ & $23.4 \pm 3.0$ & $23.1 \pm 3.3$ & 0.630 \\
\hline \multicolumn{5}{|c|}{ Etiology of limb ischemia } \\
\hline $\mathrm{TAO}, n(\%)$ & $86(83.5 \%)$ & $52(89.7 \%)$ & $34(75.6 \%)$ & 0.056 \\
\hline ASO, $n(\%)$ & $10(9.7 \%)$ & $3(5.2 \%)$ & $7(12.1 \%)$ & 0.078 \\
\hline Collagen disease, $n(\%)$ & $4(3.9 \%)$ & $2(3.4 \%)$ & $2(4.4 \%)$ & 0.795 \\
\hline Crohn's disease, $n(\%)$ & $1(1.0 \%)$ & $1(1.7 \%)$ & $0(0.0 \%)$ & 0.856 \\
\hline Eosinophilia, n (\%) & $2(1.9 \%)$ & $0(0.0 \%)$ & $2(4.4 \%)$ & 0.105 \\
\hline \multicolumn{5}{|c|}{ Risk factors of cardiovascular disease } \\
\hline Hypertension, $n$ (\%) & $7(6.8 \%)$ & $2(3.4 \%)$ & $5(11.1 \%)$ & 0.125 \\
\hline Diabetes mellitus, $n$ (\%) & $7(6.8 \%)$ & $2(3.4 \%)$ & $5(11.1 \%)$ & 0.125 \\
\hline Hyperlipidemia, $n$ (\%) & $5(4.9 \%)$ & $4(6.9 \%)$ & $1(2.2 \%)$ & 0.274 \\
\hline Smoker, $n(\%)$ & $85(82.5 \%)$ & $51(87.8 \%)$ & $34(75.6 \%)$ & 0.101 \\
\hline \multicolumn{5}{|c|}{ Blood examination } \\
\hline Fibrinogen, (mg/dL) (median [IQR]) & $316.0(255.0-408.0)$ & $304.5(238.0-351.0)$ & $336.0(278.0-467.0)$ & 0.012 \\
\hline CRP, (mg/L) (median [IQR]) & $5.40(1.90-23.00)$ & $3.75(1.60-8.50)$ & $9.50(4.00-40.80)$ & 0.001 \\
\hline Glucose, (mmol/L) (median [IQR]) & $4.70(4.40-5.00)$ & $4.70(4.50-5.00)$ & $4.70(4.40-5.00)$ & 0.762 \\
\hline Creatinine, ( $\mu$ mol/L) (median [IQR]) & $72.0(64.0-83.0)$ & $73.0(67.0-83.0)$ & $70.0(63.0-76.0)$ & 0.102 \\
\hline \multicolumn{5}{|c|}{ Treatment history } \\
\hline Antiplatelet drugs, $n$ (\%) & $58(56.3 \%)$ & $34(58.6 \%)$ & $24(53.3 \%)$ & 0.592 \\
\hline Vasodilator, $n(\%)$ & $55(53.4 \%)$ & $33(56.9 \%)$ & $22(48.9 \%)$ & 0.419 \\
\hline Statins, $n(\%)$ & $4(3.9 \%)$ & $2(3.4 \%)$ & $2(4.4 \%)$ & 0.795 \\
\hline Stenting, $n(\%)$ & $10(9.7 \%)$ & $5(8.6 \%)$ & $5(11.1 \%)$ & 0.672 \\
\hline Balloon dilation, $n(\%)$ & $7(6.8 \%)$ & $4(6.9 \%)$ & $3(6.7 \%)$ & 0.963 \\
\hline Thrombolysis, n (\%) & $16(15.5 \%)$ & $8(13.8 \%)$ & $8(17.8 \%)$ & 0.580 \\
\hline Thrombectomy, $n$ (\%) & $8(7.8 \%)$ & $3(5.2 \%)$ & $5(11.1 \%)$ & 0.264 \\
\hline Bypass surgery, $n$ (\%) & $5(4.9 \%)$ & $2(3.4 \%)$ & $3(6.7 \%)$ & 0.451 \\
\hline Endarterectomy, n (\%) & $1(1.0 \%)$ & $1(1.7 \%)$ & $0(0.0 \%)$ & 0.376 \\
\hline
\end{tabular}


Table 2 Baseline characteristics of treated limbs and transplants

\begin{tabular}{|c|c|c|c|c|}
\hline & Total, $n=103$ & Responders, $n=58$ & Non-responders, $n=45$ & $P$ value \\
\hline \multicolumn{5}{|c|}{ Rutherford class, $n(\%)$} \\
\hline IV & $12(11.6 \%)$ & $5(8.6 \%)$ & $7(15.6 \%)$ & 0.277 \\
\hline V & $91(88.4 \%)$ & $53(91.4 \%)$ & $38(84.4 \%)$ & 0.277 \\
\hline \multicolumn{5}{|l|}{ Gangrene, $n(\%)$} \\
\hline Yes & $46(44.7 \%)$ & $25(43.1 \%)$ & $21(46.7 \%)$ & 0.718 \\
\hline No & $57(55.3 \%)$ & $33(56.9 \%)$ & $24(53.3 \%)$ & 0.718 \\
\hline $\mathrm{AB}^{*}$ (median $\left.[\mathrm{IQR}]\right)$ & $0.485(0.370-0.640)$ & $0.515(0.410-0.695)$ & $0.430(0.280-0.540)$ & 0.011 \\
\hline $\mathrm{TcPO}_{2}(\mathrm{mmHg})($ median $[\mathrm{IQR}])$ & $17(6-28)$ & $24(14-30)$ & $9(4-19)$ & $<0.001$ \\
\hline \multicolumn{5}{|l|}{ Highest level of arterial occlusion } \\
\hline Above the knee or elbow, $n(\%)$ & $62(60.2 \%)$ & $28(46.6 \%)$ & $34(77.8 \%)$ & 0.001 \\
\hline Common or external iliac artery, $n(\%)$ & $4(3.9 \%)$ & $1(1.7 \%)$ & $3(6.7 \%)$ & 0.198 \\
\hline Common femoral artery or axillary artery, $n(\%)$ & $5(4.8 \%)$ & $4(6.9 \%)$ & $1(2.2 \%)$ & 0.383 \\
\hline Superficial femoral artery or brachial artery, $n(\%)$ & $36(35.0 \%)$ & $15(25.9 \%)$ & $21(46.7 \%)$ & 0.037 \\
\hline Popliteal artery, $n$ (\%) & $17(16.5 \%)$ & $7(12.1 \%)$ & $10(22.2 \%)$ & 0.169 \\
\hline Below the knee or elbow, $n(\%)$ & $41(39.8 \%)$ & $31(53.4 \%)$ & $10(22.2 \%)$ & 0.001 \\
\hline Artery in the calf or forearm, $n(\%)$ & $28(27.2 \%)$ & $23(39.7 \%)$ & $5(11.1 \%)$ & 0.001 \\
\hline Artery below the ankle or wrist, $n(\%)$ & $13(12.6 \%)$ & $8(13.7 \%)$ & $5(11.1 \%)$ & 0.684 \\
\hline \multicolumn{5}{|c|}{ Type of transplantation } \\
\hline Purified for CD34 ${ }^{+}$cells, $n(\%)$ & $52(50.5 \%)$ & $30(51.7 \%)$ & $22(48.9 \%)$ & 0.775 \\
\hline Not purified, $n(\%)$ & $51(49.5 \%)$ & $28(48.3 \%)$ & $23(51.1 \%)$ & 0.775 \\
\hline Total transplanted CD34+ cells (million/L) (median [IQR]) & $43.0(26.0-84.0)$ & $51.5(26.0-103.0)$ & $40.0(26.0-68.0)$ & 0.114 \\
\hline Cell viability (median [IQR]) & $98.4 \%(97.7 \%-98.6 \%)$ & $98.3 \%(97.5 \%-98.6 \%)$ & $98.4 \%(98.2 \%-98.6 \%)$ & 0.212 \\
\hline
\end{tabular}

${ }^{*}$ ABls of 99 patients with lower limbs treated were included in this analysis, while the other 4 patients with upper limbs treated were excluded $\mathrm{IQR}$, interquartile range; $\mathrm{ABl}$, ankle-brachial index; $\mathrm{TCPO}_{2}$ : transcutaneous pressure of oxygen; $\mathrm{CD}$, cluster of differentiation
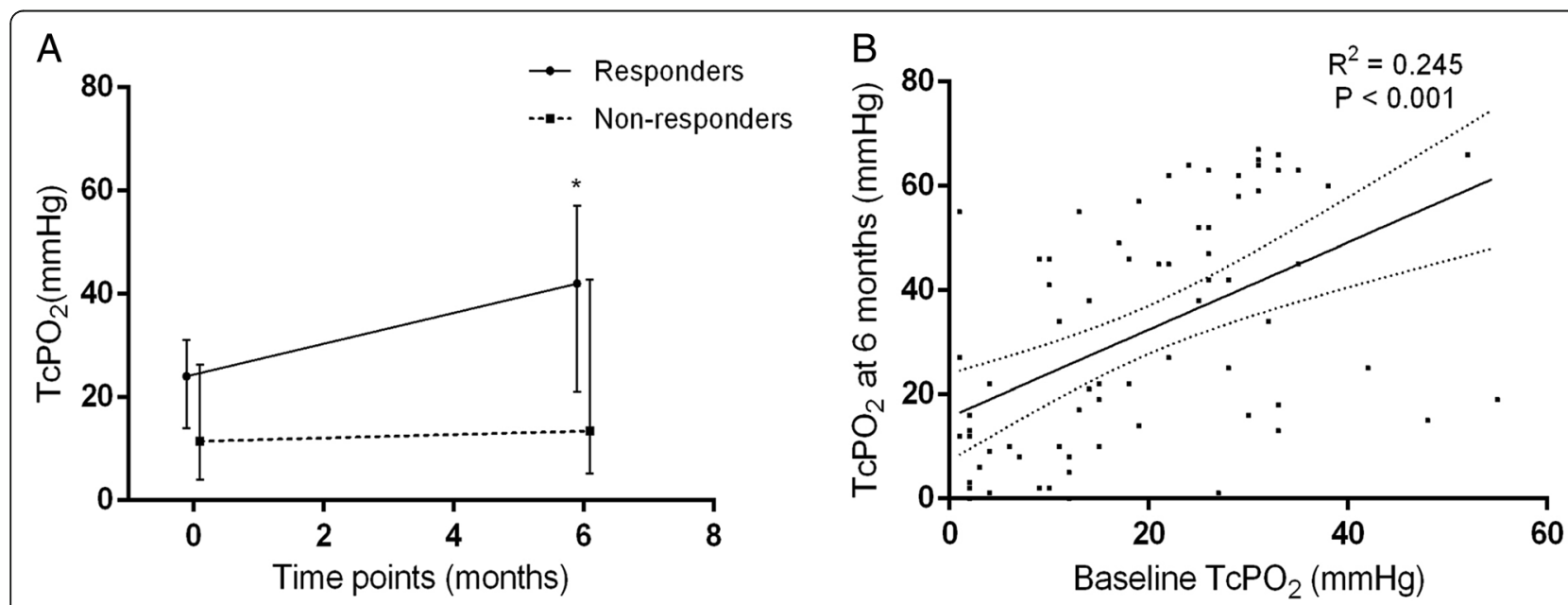

Fig. 2 Comparison of longitudinal $\mathrm{TCPO}_{2}$ changes (median with interquartile range) in the ischemic limbs of the responders and non-responders (a) and linear regression between $\mathrm{TCPO}_{2}$ at baseline and at 6 months post-transplantation, depicted with a solid fitting line and dotted $95 \%$ confidential interval bars (b). *The difference between 6 months and baseline was statistically significant $(P<0.05)$. TcPO ${ }_{2}$, transcutaneous pressure of oxygen 
0.775) (Table 2). The total transplanted $\mathrm{CD}^{+} 4^{+}$cell count appeared to be higher in the responder group (median $51.5 \times 10^{6}$, IQR $26.0-103.0 \times 10^{6}$ ) than in the non-responder group (median $40.0 \times 10^{6}$, IQR 26.0$\left.68.0 \times 10^{6}\right)$, although the difference was not statistically significant $(P=0.114)$ (Table 2$)$.

\section{Prognostic factors}

According to the results of the univariate logistic regression, several variables were screened out: age $\geq 50$ years [odds ratio (OR) 0.276 , 95\% confidence interval (CI) 0.118-0.647, $P$ $=0.003], \mathrm{CRP}>3 \mathrm{mg} / \mathrm{L}($ OR $0.287,95 \%$ CI $0.117-0.702, P$ $=0.006)$, fibrinogen $>4 \mathrm{~g} / \mathrm{L}(\mathrm{OR} 0.172,95 \%$ CI 0.064-0.459, $P<0.001$ ), ASO (OR 0.296, 95\% CI 0.072-1.218, $P=0.092$ ), highest arterial occlusion level above the knee or elbow (OR 0.249, 95\% CI 0.104-0.595, $P=0.002$ ), ABI (OR 8.345, 95\% CI 1.301-53.54, $P=0.025), \mathrm{TcPO}_{2}$ (OR 1.069, 95\% CI1.030-1.109, $P<0.001$ ), and base-10 logarithm (Log) of total transplanted CD34 ${ }^{+}$cell count (OR 2.274, 95\% CI $0.850-6.079, P=0.102$ ) (Table 3). The linear regression analysis showed a significant correlation between the levels of CRP and fibrinogen $\left(R^{2}=0.376, P<0.001\right)$, and the correlation between ASO and age was also significant according to Pearson's chi-square test $(P<0.001)$ (Additional file 1 ).

Age $\geq 50$ years was chosen for further multivariate regression prior to the etiology (ASO), and the reason is presented in the discussion section. ABI was also removed before the multivariate regression because it could only be examined in the lower limbs $(n=99$ in our study) and was not suitable for a prognostic analysis of the patients with upper limb ischemia. Either CRP or fibrinogen could be incorporated into the multivariate regression. When incorporating fibrinogen $>4 \mathrm{~g} / \mathrm{L}$, the multivariate regression finally indicated that age $\geq 50$ years (OR $0.201,95 \%$ CI $0.068-0.595, P=0.004$ ), fibrinogen $>4 \mathrm{~g} / \mathrm{L}$ (OR 0.176, 95\% CI 0.055-0.563, $P=0.003$ ), highest arterial occlusion level above the knee or elbow (OR 0.232, 95\% CI 0.077-0.703, $P=0.010), \mathrm{TcPO}_{2}(\mathrm{OR}$ 1.062, 95\% CI 1.017-1.110, $P=0.006)$, and Log total transplanted CD34 ${ }^{+}$cell count (OR 3.506, 95\% CI 1.02112.039, $P=0.046)$ were independent prognostic factors of responders (Table 3 ). The regression model was statistically significant $\left(\chi^{2}=44.585, P<0.001\right)$ and explained $47.1 \%$ (Negelkerke $R^{2}$ ) of the variance in the outcome. When incorporating CRP $>3 \mathrm{mg} / \mathrm{L}$, the ORs of the other prognostic factors remained significant, but the Negelkerke $R^{2}$ dropped to $42.9 \%$ (Additional file 1 ). Thus, the prior model incorporating fibrinogen $>4 \mathrm{~g} / \mathrm{L}$ was chosen for the development of the nomogram.

\section{Nomogram and validation}

Based on the results of the multivariate regression, a nomogram incorporating five predictors was established to predict the probability of responders (Fig. 3). In the

Table 3 Univariate and multivariate logistic regression analysis of prognostic factors

\begin{tabular}{|c|c|c|c|c|}
\hline \multirow[t]{2}{*}{ Candidate variable } & \multicolumn{2}{|l|}{ Univariate analysis } & \multicolumn{2}{|l|}{ Multivariate analysis } \\
\hline & $\mathrm{OR}(95 \% \mathrm{Cl})$ & $P$ value & OR $(95 \% \mathrm{Cl})$ & $P$ value \\
\hline Age $\geq 50$ years & $0.276(0.118-0.647)$ & 0.003 & $0.201(0.068-0.595)$ & 0.004 \\
\hline Body mass index $\left(\mathrm{kg} / \mathrm{m}^{2}\right)$ & $1.032(0.909-1.172)$ & 0.627 & & \\
\hline Diabetes mellitus & $0.286(0.053-1.547)$ & 0.146 & & \\
\hline Hypertension & $0.286(0.053-1.547)$ & 0.146 & & \\
\hline Hyperlipidemia & $3.259(0.351-30.22)$ & 0.298 & & \\
\hline Smoker & $2.357(0.831-6.684)$ & 0.107 & & \\
\hline $\mathrm{CRP}>3 \mathrm{mg} / \mathrm{L}$ & $0.287(0.117-0.702)$ & 0.006 & & \\
\hline Fibrinogen $>4 \mathrm{~g} / \mathrm{L}$ & $0.172(0.064-0.459)$ & $<0.001$ & $0.176(0.055-0.563)$ & 0.003 \\
\hline Etiology $=$ ASO & $0.296(0.072-1.218)$ & 0.092 & & \\
\hline Rutherford class $=\mathbb{I V}$ & $0.512(0.151-1.736)$ & 0.283 & & \\
\hline Gangrene & $0.866(0.396-1.894)$ & 0.718 & & \\
\hline Arterial occlusion above the knee or elbow* & $0.249(0.104-0.595)$ & 0.002 & $0.232(0.077-0.703)$ & 0.010 \\
\hline$\left.A B\right|^{* *}$ & $8.345(1.301-53.54)$ & 0.025 & & \\
\hline $\mathrm{TCPO}_{2}(\mathrm{mmHg})$ & 1.069 (1.030-1.109) & $<0.001$ & $1.062(1.017-1.110)$ & 0.006 \\
\hline Using purified $\mathrm{CD} 34^{+}$cells & $0.913(0.425-1.961)$ & 0.816 & & \\
\hline Log (total transplanted CD34+ cell counts) & $2.274(0.850-6.079)$ & 0.102 & $3.506(1.021-12.039)$ & 0.046 \\
\hline
\end{tabular}

*Defined as the highest occlusion level located at common iliac artery, external iliac artery, common femoral artery, superficial femoral artery, popliteal artery, axillary artery, or brachial artery

**ABls of 99 patients with lower limbs treated were included in this analysis, while the other 4 patients with upper limb lesions were excluded

*** Base-10 logarithm of total transplanted $\mathrm{CD}_{3} 4^{+}$cell counts

$\mathrm{OR}$, odds ratio; $\mathrm{Cl}$, confidential interval; $\mathrm{CRP}, \mathrm{C}$-reaction protein; $\mathrm{ASO}$, atherosclerosis obliterans; $\mathrm{ABI}$, ankle-brachial index; $\mathrm{TcPO}_{2}$ : transcutaneous pressure of oxygen; $C D$, cluster of differentiation 


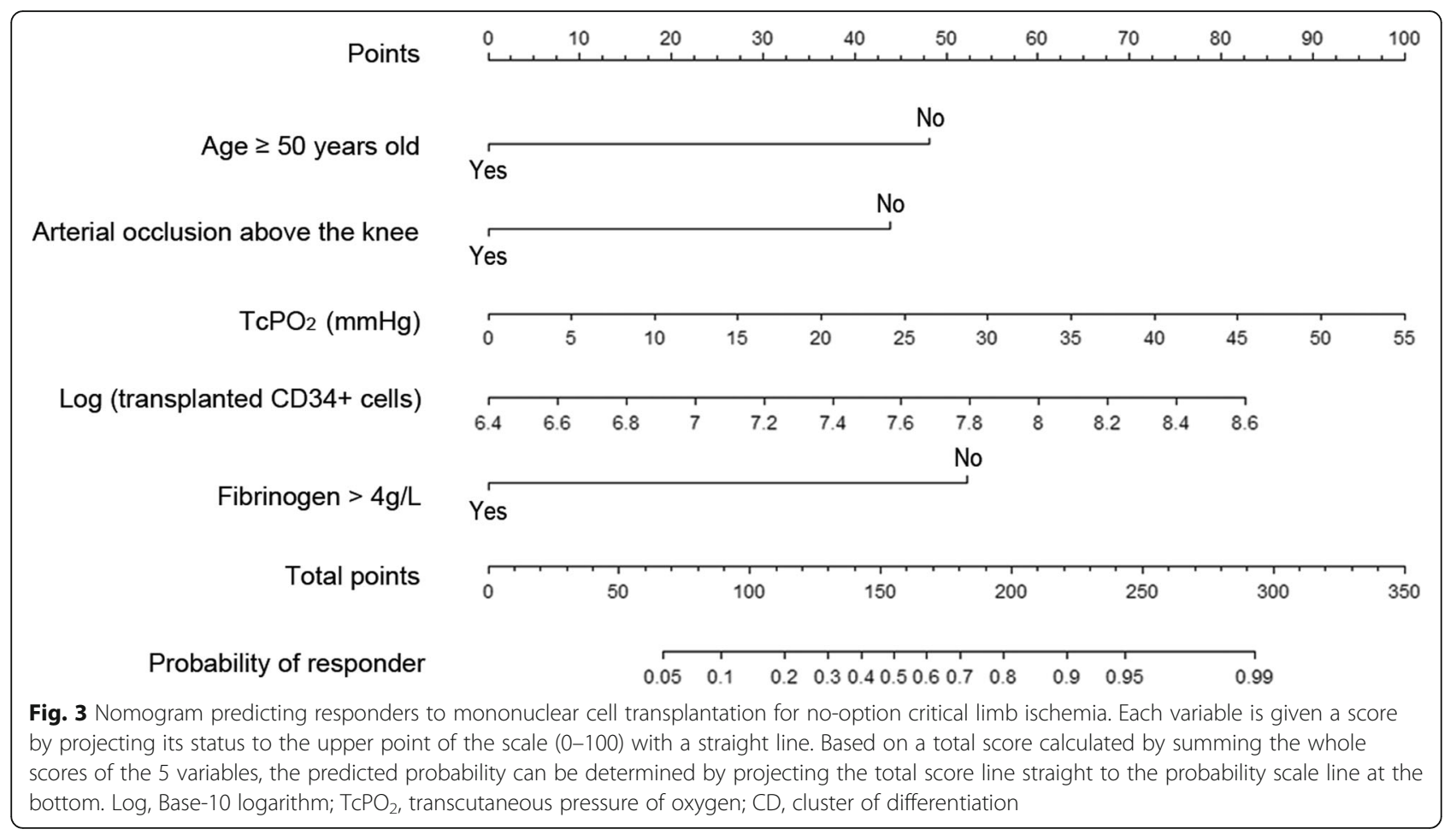

nomogram, each variable was given a score by projecting its status to the upper point scale (0-100) with a straight line. A total score was then calculated by summing up the whole scores of the five variables. The predicted probability of a responder could be determined by projecting the total score line straight to the probability scale line in the bottom (Fig. 3). A ROC curve was generated to address the sensitivity and specificity of the nomogram. The area under the ROC curve was 0.851 (95\% CI 0.779-0.922), indicating good discrimination for responders (Fig. 4a). With 1000 cycles of bootstrap resampling, the bias-corrected calibration curve was close to the $45^{\circ}$ line, indicating good agreement between the predicted probability and the observed probability of responders (Fig. 4b). The typical CTA and longitudinal foot photos of a responder and a
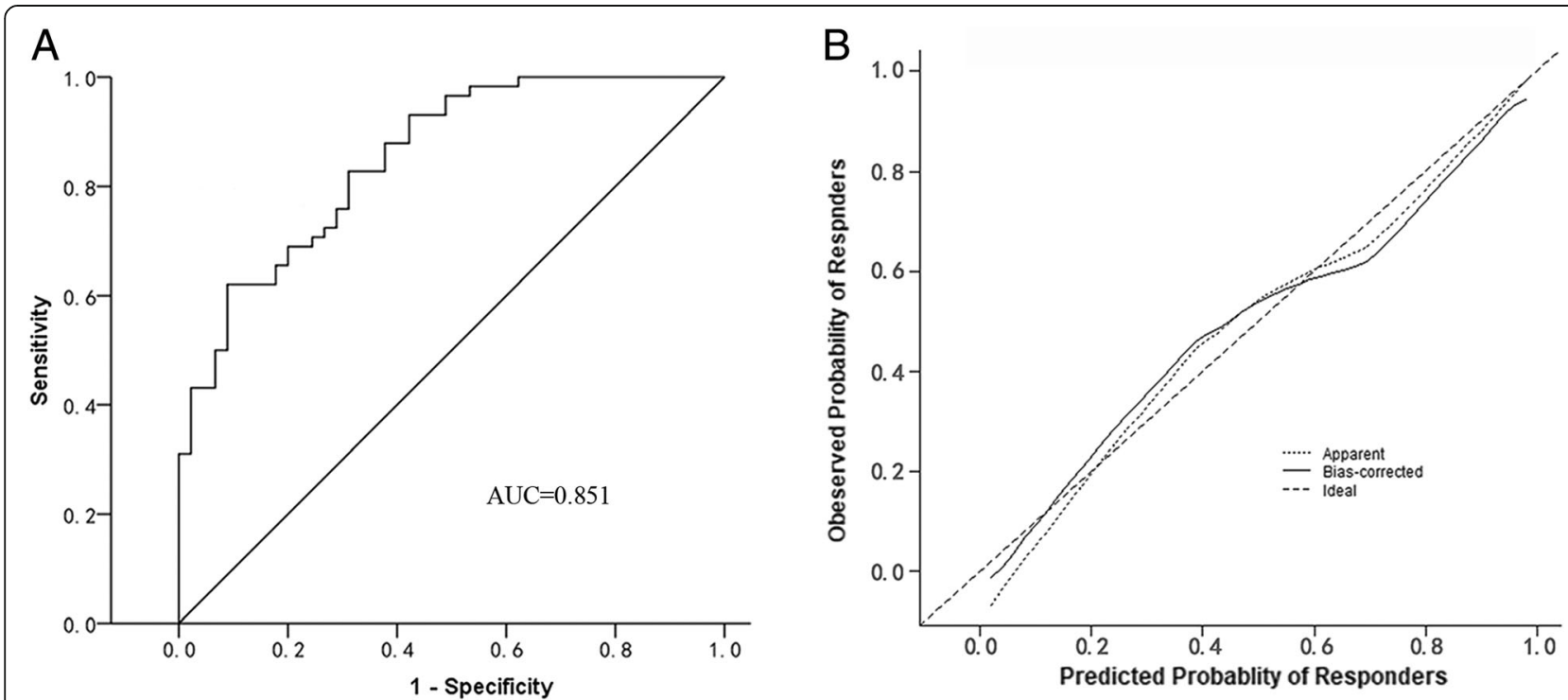

Fig. 4 Receiver operating characteristic (ROC) curve to address the sensitivity and specificity of the nomogram, with an area under the curve of 0.851 (a) and bias-corrected calibration curve with 1000 cycles of bootstrap resampling to indicate good agreement between the predicted probability and the observed probability of responders (b). AUC, area under the curve 
non-responder according to the nomogram were shown in Fig. 5.

\section{Discussion}

Summary of the results

The current study investigated the prognostic factors related to the therapeutic efficacy of MNC transplantation in treating NO-CLI, and, to our knowledge, this study has initiatively developed a visualized scale to provide an individualized prediction of whether a patient would respond to MNC transplantation. Our results revealed that age $\geq 50$ years, blood fibrinogen $>4 \mathrm{~g} / \mathrm{L}$, and arterial occlusion above the knee/elbow were negative factors in terms of the probability of being a responder, while the $\mathrm{TcPO}_{2}$ value and total transplanted $\mathrm{CD} 34^{+}$cell count positively correlated with the probability of being a responder. These prognostic factors were independent from each other, and based on these factors, we built a predictive nomogram with high discrimination and accuracy.

\section{Prognostic factors associated with patients}

Patient age has been considered a critical factor that affects the prognosis after cell therapy. Madaric et al.'s study identified age as an independent predictor for the 1-year amputation-free survival of CLI patients who received MNC transplantation [21]. In our study, we found that age $\geq 50$ years was an independent negative prognostic factor for responders who achieved complete CLI remission within 6 months. The critical role of age in the prognosis might be explained with several aspects. First, auto-transplants from elderly patients might not induce sufficient angiogenesis or vasculogenesis due to the aging-related impairment of the survival, migration, differentiation, and paracrine ability of the pro-angiogenic cell fraction [22-24]. Li et al. demonstrated that the ages of human donors correlated inversely with the angiogenic potency of mononuclear cells in preclinical experiments [17]. Accordingly, the aging-related impairment of transplants might explain the inconsistent prognoses of the population at various ages. Second, the relatively poor general condition of elderly patients, complicated

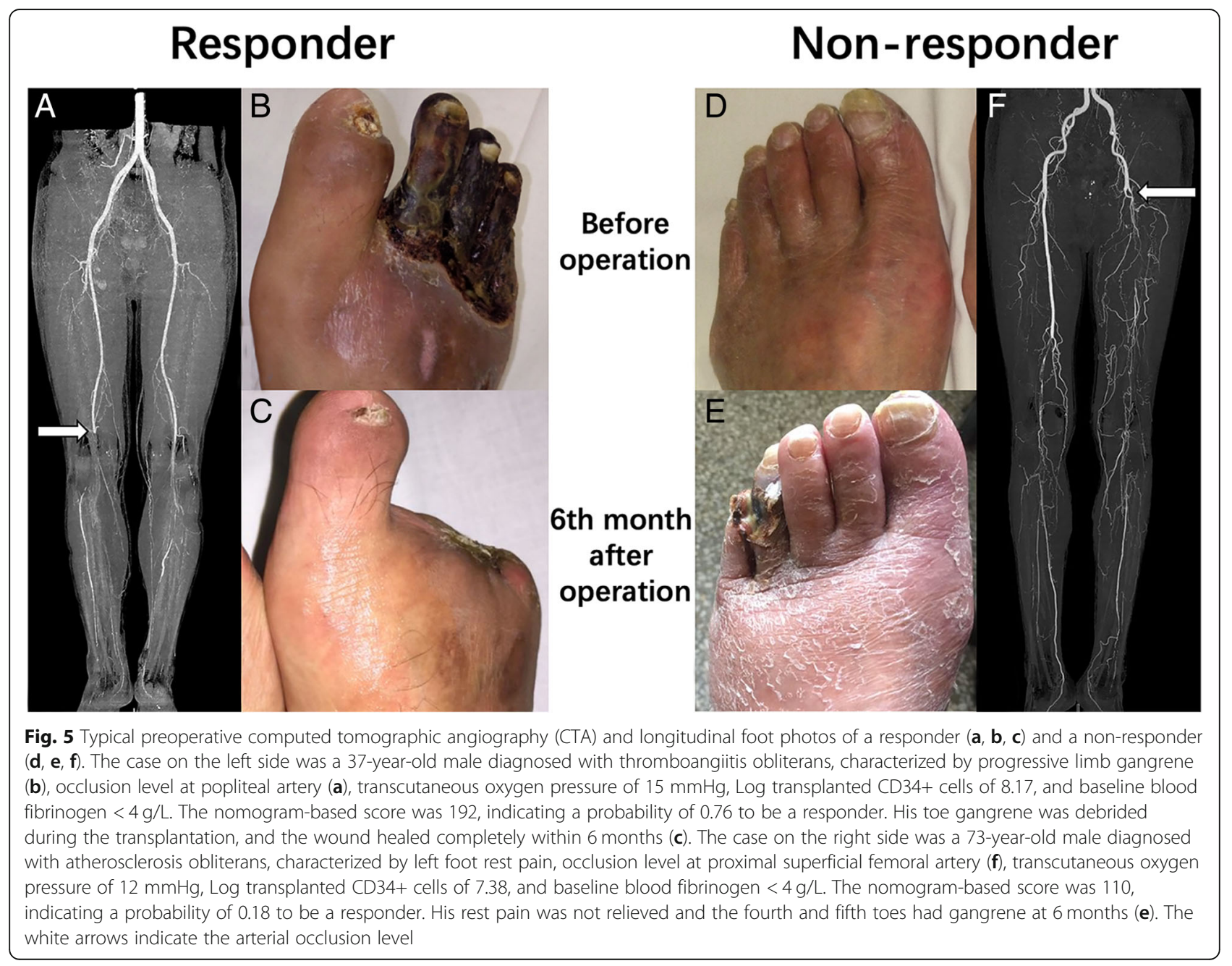


by high risks of organ dysfunction or cardiovascular diseases, was likely to reduce the probability of overall survival and amputation-free survival. However, this notion might not explain the age-related CLI remission in our study because the time to endpoint was relatively short and because only one death event occurred, and the enrolled patients were generally in good condition with low frequencies of severe complications. Previous studies have also suggested that the etiology of CLI is a critical prognostic factor. The Therapeutic Angiogenesis by Cell Transplantation (TACT) trial reported that TAO patients who received BM-MNC transplantation had significantly higher major amputation-free rates (1 year 93.2\%; 10 years $87.9 \%$ ) than ASO patients ( 1 year $78.7 \%$; 10 years $70.1 \%$ ). The PROVASA trial suggested that TAO patients responded to the MNC therapy more favorably than ASO patients, with a higher probability of rest pain alleviation and ulcer healing [11]. Likewise, our study observed that ASO was associated with a reduced, though insignificant, probability of being a responder. Because the patients with TAO were characterized by a much younger onset age than those with ASO, which was also confirmed in our study, we speculate that age and aging-related cell functional impairments at least partially underlie the relevance of etiology to prognosis. Therefore, age $\geq 50$ years was chosen for the multiple regression and nomogram prior to the etiology of ASO, because the former was considered to have a more direct impact on the impaired therapeutic efficacy of MNC transplantation than the latter.

The morphology of the arterial lesion has been considered critical to endovascular and bypass surgery. In contrast, most of the previous clinical studies of cell therapy did not take the anatomical property of the involved arteries into consideration. A recent study, however, found that CLI patients without amputations at 3 months post-MNC transplantation showed more favorable patency of the upper-popliteal arteries than those with major amputations [25]. Our results further indicated that arterial occlusion above the knee or elbow was a significant negative predictor for responders and was independent of age. Notably, in most non-responders $(77.8 \%)$, the occlusion level involved the popliteal or upper-popliteal artery. We speculate that the above-the-knee patency of the lower limb arteries can bring more favorable blood perfusion to the transplanted site (calf and distal foot), thus enhancing the tissue regeneration induced by cell therapy. Accordingly, we suggest that a sufficient inflow from the upper-popliteal arteries to the calf might be a necessary condition for MNC transplantation. In addition, the significant inverse correlation between the above-the-knee occlusion and responders might partially explain the poor prognosis of cell therapy in ASO patients with CLI because the upper-popliteal arteries, especially the superficial femoral artery, were mostly affected in ASO, while TAO was characterized by the initial invasion of distal small arteries below the knee.

$\mathrm{TcPO}_{2}$ represents the tension of oxygen disseminated from subcutaneous microcirculation, which reflects distal peripheral perfusion. The $\mathrm{TcPO}_{2}$ measurement has been considered a useful predictor for post-debridement chronic ischemic ulcer healing, with a threshold value of $20-40 \mathrm{mmHg}$, and a $\mathrm{TcPO}_{2}$ value over $40 \mathrm{mmHg}$ seemed to promise complete ulcer healing [26]. In our study, the responders had significantly higher baseline $\mathrm{TcPO}_{2}$ values for the distal foot (median $24 \mathrm{mmHg}$ ) than the non-responders (median $9 \mathrm{mmHg}$ ). The multivariate regression analysis also showed that the baseline $\mathrm{TcPO}_{2}$ value was an independent prognostic factor, which was consistent with several previous studies $[21,25]$. According to the longitudinal data, we further found that the responders achieved a significant improvement in $\mathrm{TcPO}_{2} 6$ months post-transplantation, while the non-responders did not. Moreover, the $\mathrm{TcPO}_{2}$ at baseline correlated significantly with that at 6 months. These results suggested that the restoration of peripheral perfusion induced by MNC transplantation was characterized by a favorable baseline condition of local microcirculation, which might explain the predictive role of baseline $\mathrm{TcPO}_{2}$.

Inflammatory biomarkers such as fibrinogen, CRP, and interleukin- 6 have recently been considered critical risk factors for the development and progression of PAD [27]. Our study has demonstrated that responders to the MNC transplantation are characterized by significantly lower baseline values of both CRP and fibrinogen than non-responders, and these two biomarkers are significantly correlated with each other according to the linear regression analysis. Because both biomarkers are acute-phase reactant proteins that are produced by the liver during inflammation, we suggest that the systemic or local inflammatory reaction can negatively influence the therapeutic angiogenesis of the MNC transplant. On the one hand, inflammatory factors such as CRP can directly inhibit EPC survival, differentiation, and angiogenic potency [28]. Fibrinogen also plays a leading role in thrombosis, and elevated fibrinogen can increase the cumulative rates of ischemic events in the course of PAD [29, 30]. Our results revealed that both fibrinogen $>4 \mathrm{~g} / \mathrm{L}$ and $\mathrm{CRP}>3 \mathrm{mg} / \mathrm{L}$ can be predictors that are independent of the other factors. The former was chosen to develop the nomogram only because it improved the fitting goodness of the model relative to the latter. More studies are required to investigate the most appropriate inflammatory biomarker to predict the prognosis of CLI candidates for cell therapy. 


\section{Prognostic factors associated with the transplants}

In addition to the patient's clinical background, the characteristics of the transplants had a critical influence on the prognosis. CD34 $4^{+}$cells represent a main subset of stem/progenitor cells in PBMNC transplants that potentiate neovascularization in the ischemic area [31]. The number of transplanted human $\mathrm{CD}_{3} 4^{+}$cells appeared to have a strong correlation with the blood perfusion index in the severe combined immunodeficiency mouse model [23]. In addition, the increased number of transplanted $\mathrm{CD}_{3} 4^{+}$cells improved the chance of wound healing [32]. Previous clinical studies suggested thatCD $34^{+}$cells should be used as quality control for transplants, and a total $\mathrm{CD} 34^{+}$cell count over $10^{5} / \mathrm{kg}$ body weight seemed appropriate [33]. Our study further demonstrated that the Log total transplanted $\mathrm{CD} 34^{+}$cell count was an independent positive predictor of responders, indicating a cell dose-dependent therapeutic efficacy.

On the other hand, we found that responders at 6 months were not associated with transplant purification for $\mathrm{CD}_{34}{ }^{+}$cells or the use of non-purified MNCs. This is consistent with our recent published 12-month results of a prospective randomized trial comparing these two types of cells treating NO-CLI [34]. Accordingly, we believe that both a specific $\mathrm{CD}_{3}{ }^{+}$cell-based and mixed cell-based MNC transplantations might be feasible for NO-CLI patients. A longer-term follow-up study will be needed to compare the advantages and disadvantages of these two transplantation types.

\section{Feasibility of the nomogram}

This originally developed nomogram for predicting complete remissions from NO-CLI at 6 months post-MNC transplantation was based on a consecutive cohort of 110 patients (103 ultimately analyzed) in a single center. The nomogram had a good discriminative ability and predictive accuracy in the validation with the ROC and calibration curves. Notably, our patient population was characterized by a relatively young age and a low frequency of cardiovascular risk and organ dysfunction and was exclusively classified as Rutherford class 4-5. In other studies based on elderly populations with advanced limb ischemia and high risks of systemic diseases, factors such as a history of hemodialysis, Rutherford classification of 6 , and high blood glucose also appeared to be relevant to poor prognosis after MNC therapy [16, 35, 36]. Thus, we suggest that our nomogram should not be applied to very elderly individuals with advanced CLI (Rutherford class 6) or a poor general condition to avoid wide uncertainty of the prediction.

\section{Study limitations}

There were several limitations to the current study. First, the current study was retrospectively designed based on a single-centered consecutive cohort, and there was no parallel placebo control group due to ethical concerns. Additionally, the sample size was not large enough for the evaluation of more specific variables, such as cell function and viability. Second, our study focused on short-term responders to MNC transplantation rather than long-term remission from CLI, which is also the target of therapeutic angiogenesis. Therefore, a prolonged follow-up of the cohort is needed in the future to confirm the robustness of the identified prognostic factors. Third, due to the nature of the single-centered design and relatively small sample size of the study, an external validation of the nomogram was not performed, and the generalizability of this predictive tool remains to be examined in cohorts from other centers.

\section{Conclusion}

Age $\geq 50$ years, blood fibrinogen $>4 \mathrm{~g} / \mathrm{L}$, and arterial occlusion above the knee/elbow were independent negative prognostic factors, while baseline $\mathrm{TcPO}_{2}$ and the Log transplanted $\mathrm{CD} 34^{+}$cell count were independent positive prognostic factors for responders who survived and achieved complete remission from CLI 6 months post-MNC transplantation. Accordingly, a nomogram with high discrimination and accuracy was developed to provide individualized predictions. A prolonged follow-up study of this cohort is needed to evaluate the robustness of these predictors, and the generalizability of the nomogram remains to be examined in cohorts from other centers.

\section{Additional file}

Additional file 1 Supplementary material. (DOCX $351 \mathrm{~kb}$ )

\begin{abstract}
Abbreviations
ABI: Ankle-brachial index; ASO: Arteriosclerosis obliterans; BM: Bone marrow; $\mathrm{Cl}$ : Confidence interval; CLI: Critical limb ischemia; IQR: Interquartile range; MNC: Mononuclear cell; NO-CLI: No-option critical limb ischemia; OR: Odds ratio; PAD: Peripheral artery disease; PB: Peripheral blood; ROC: Receiver operating characteristic; SD: Standard deviation; TAO: Thromboangiitis obliterans; $\mathrm{TCPO}_{2}$ : Transcutaneous pressure of oxygen
\end{abstract}

\section{Acknowledgements}

We would also like to thank Dr. Farrell Owen Mendelsohn from the Center for Therapeutic Angiogenesis, Birmingham (Alabama, USA), Dr. Atsuhiko Kawamoto from Division of Vascular Regeneration Therapy, Laboratory for Stem Cell Translational Research, Institute of Biomedical Research and Innovation, Kobe, Japan, Dr. Satoaki Matoba from Department of Cardiovascular Medicine, Kyoto Prefectural University of Medicine, Kyoto, Japan, and Dr. Douglas W. Losordo from Northwestern University, Chicago, USA, for their sincere help during the early years when we started PCC transplantation. 


\section{Funding}

This research was supported by the Municipal Human Resources Development Program for Outstanding Leaders in Medical Disciplines in Shanghai (Grant No. 2018BR40) and China National Natural Science Funds (Grant No. 30801122). And Zhongshan Funds for the Institute of Vascular Surgery, Fudan University, China. And Youth Science Fund of Zhongshan Hospital, Fudan University, Shanghai, China (2018ZSQN15).

\section{Availability of data and materials}

The datasets used and analyzed during the current study are available from the corresponding author on reasonable request

\section{Authors' contributions}

PT and LH designed the study, wrote the manuscript, and analyzed the patient data. DZ and FW designed the study and wrote the manuscript. LYF, $L Y, F G, C B, J J, Y J, X X, G D, Z T, S Y, S Z, L P, W Z$, and GS wrote the manuscript and collected the data of the patients. All authors read and approved the final manuscript.

\section{Ethics approval and consent to participate}

The study design was approved by the Ethics Committee of Zhongshan Hospital, Fudan University, Shanghai, China. All included patients were informed about the nature of the study and gave their written informed consent.

\section{Consent for publication}

All patients signed a consent form for their data to be used for research or publication.

\section{Competing interests}

The authors declare that they have no competing interests.

\section{Publisher's Note}

Springer Nature remains neutral with regard to jurisdictional claims in published maps and institutional affiliations.

\section{Author details}

'Department of Vascular Surgery, Zhongshan Hospital, Fudan University, Shanghai 200032, China. ${ }^{2}$ Institute of Vascular Surgery, Fudan University, Shanghai, China. ${ }^{3}$ Department of Hematology, Zhongshan Hospital, Fudan University, Shanghai, China.

Received: 12 November 2018 Revised: 10 December 2018 Accepted: 19 December 2018 Published online: 11 January 2019

\section{References}

1. Ryu HM, Kim JS, Ko YG, Hong MK, Jang Y, Choi DH. Comparison of clinical outcome of infrapopliteal angioplasty between Korean diabetic and nondiabetic patients with critical limb ischemia. Circ J. 2012;76:335-41.

2. Norgren L, Hiatt WR, Dormandy JA, Nehler MR, Harris KA, Fowkes FG. Inter society consensus for the Management of Peripheral Arterial Disease (TASC II). J VascSurg. 2007:45(SupplS):S5-67.

3. Siracuse JJ, Menard MT, Eslami MH, et al. Comparison of open and endovascular treatment of patients with critical limb ischemia in the Vascular Quality Initiative. J VascSurg. 2016;63:958-65 e1.

4. Lawall H, Bramlage P, Amann B. Treatment of peripheral arterial disease using stem and progenitor cell therapy. J VascSurg. 2011:53:445-53.

5. Horie T, Yamazaki $S$, Hanada $S$, et al. Outcome from a randomized controlled clinical trial- improvement of peripheral arterial disease by granulocyte Colony-stimulating factor-mobilized autologous peripheralblood-mononuclear cell transplantation (IMPACT). Circ J. 2018;82:2165-74.

6. Pignon B, Sevestre MA, Kanagaratnam L, et al. Autologous bone marrow mononuclear cell implantation and its impact on the outcome of patients with critical limb ischemia- results of a randomized, double-blind, placebocontrolled trial. Circ J. 2017:81:1713-20.

7. Teraa M, Sprengers RW, Schutgens RE, et al. Effect of repetitive intra-arterial infusion of bone marrow mononuclear cells in patients with no-option limb ischemia: the randomized, double-blind, placebo-controlled rejuvenating endothelial progenitor cells via transcutaneous intra-arterial supplementation (JUVENTAS) trial. Circulation. 2015;131:851-60.
8. Dong Z, Chen B, Fu W, et al. Transplantation of purified CD34+ cells in the treatment of critical limb ischemia. J Vasc Surg. 2013;58:404-11 e3.

9. Dubsky M, Jirkovska A, Bem R, et al. Both autologous bone marrow mononuclear cell and peripheral blood progenitor cell therapies similarly improve ischaemia in patients with diabetic foot in comparison with control treatment. Diabetes Metab Res Rev. 2013;29:369-76.

10. Losordo DW, Kibbe MR, Mendelsohn F, et al. A randomized, controlled pilot study of autologous CD34+ cell therapy for critical limb ischemia. Circ Cardiovasc Interv. 2012:5:821-30

11. Walter $\mathrm{DH}$, Krankenberg $\mathrm{H}$, Balzer JO, et al. Intraarterial administration of bone marrow mononuclear cells in patients with critical limb ischemia: a randomized-start, placebo-controlled pilot trial (PROVASA). Circ Cardiovasc Interv. 2011:4:26-37.

12. Prochazka V, Gumulec J, Jaluvka F, et al. Cell therapy, a new standard in management of chronic critical limb ischemia and foot ulcer. Cell Transplant. 2010;19:1413-24.

13. Tateishi-Yuyama E, Matsubara $\mathrm{H}$, Murohara $T$, et al. Therapeutic angiogenesis for patients with limb ischaemia by autologous transplantation of bone-marrow cells: a pilot study and a randomised controlled trial. Lancet. 2002;360:427-35.

14. Szabo GV, Kovesd Z, Cserepes J, Daroczy J, Belkin M, Acsady G. Peripheral blood-derived autologous stem cell therapy for the treatment of patients with late-stage peripheral artery disease-results of the short- and long-term follow-up. Cytotherapy. 2013;15:1245-52.

15. Ozturk A, Kucukardali Y, Tangi F, et al. Therapeutical potential of autologous peripheral blood mononuclear cell transplantation in patients with type 2 diabetic critical limb ischemia. J Diabetes Complicat. 2012;26:29-33.

16. Kondo K, Yanishi K, Hayashida R, et al. Long-term clinical outcomes survey of bone marrow-derived cell therapy in critical limb ischemia in Japan. Circ J. 2018:82:1168-78

17. Li TS, Kubo M, Ueda $K$, et al. Identification of risk factors related to poor angiogenic potency of bone marrow cells from different patients. Circulation. 2009:120:S255-61.

18. Iwasaki H, Kawamoto A, Ishikawa M, et al. Dose-dependent contribution of CD34-positive cell transplantation to concurrent vasculogenesis and cardiomyogenesis for functional regenerative recovery after myocardial infarction. Circulation. 2006;113:1311-25.

19. Pan T, Wei Z, Fang Y, Dong Z, Fu W. Therapeutic efficacy of CD34(+) cell-involved mononuclear cell therapy for no-option critical limb ischemia: a meta-analysis of randomized controlled clinical trials. Vasc Med. 2018;23:219-31.

20. Rigato M, Monami M, Fadini GP. Autologous cell therapy for peripheral arterial disease: systematic review and meta-analysis of randomized nonrandomized, and noncontrolled studies. Circ Res. 2017;120:1326-40.

21. Madaric J, Klepanec A, Valachovicova M, et al. Characteristics of responders to autologous bone marrow cell therapy for no-option critical limb ischemia. Stem Cell Res Ther. 2016:7:116.

22. Kubo M, Li TS, Kurazumi H, et al. Hypoxic preconditioning enhances angiogenic potential of bone marrow cells with aging-related functiona impairment. Circ J. 2012;76:986-94.

23. Li TS, Kubo M, Ueda K, Murakami M, Mikamo A, Hamano K. Impaired angiogenic potency of bone marrow cells from patients with advanced age, anemia, and renal failure. J Thorac Cardiovasc Surg. 2010;139:459-65.

24. Dimmeler S, Leri A. Aging and disease as modifiers of efficacy of cell therapy. Circ Res. 2008:102:1319-30.

25. Aoyama N, Nishinari M, Ohtani S, et al. Clinical features and predictors of patients with critical limb ischemia who responded to autologous mononuclear cell transplantation for therapeutic angiogenesis. Heart Vessel. 2017;32:1099-108

26. Ruangsetakit C, Chinsakchai $\mathrm{K}$, Mahawongkajit $\mathrm{P}$, Wongwanit C, Mutirangura P. Transcutaneous oxygen tension: a useful predictor of ulcer healing in critical limb ischaemia. J Wound Care. 2010;19:202-6.

27. Hazarika S, Annex BH. Biomarkers and genetics in peripheral artery disease. Clin Chem. 2017:63:236-44.

28. Verma S, Kuliszewski MA, Li SH, et al. C-reactive protein attenuates endothelial progenitor cell survival, differentiation, and function: further evidence of a mechanistic link between C-reactive protein and cardiovascular disease. Circulation. 2004;109:2058-67.

29. Altes P, Perez P, Esteban C, et al. Raised fibrinogen levels and outcome in outpatients with peripheral artery disease. Angiology. 2018;69:507-12.

30. Grenon SM, Cohen BE, Smolderen K, Vittinghoff E, Whooley MA, Hiramoto J. Peripheral arterial disease, gender, and depression in the heart and soul study. J Vasc Surg. 2014;60:396-403. 
31. Schatteman GC, Hanlon HD, Jiao C, Dodds SG, Christy BA. Blood-derived angioblasts accelerate blood-flow restoration in diabetic mice. J Clin Invest. 2000;106:571-8

32. Tanaka R, Masuda H, Fujimura S, et al. Quality-quantity control culture enhances Vasculogenesis and wound healing efficacy of human diabetic peripheral blood CD34+ cells. Stem Cells Transl Med. 2018;7:428-38.

33. Kawamoto A, Katayama M, Handa N, et al. Intramuscular transplantation of G-CSF-mobilized CD34(+) cells in patients with critical limb ischemia: a phase I/lla, multicenter, single-blinded, dose-escalation clinical trial. Stem Cells. 2009;27:2857-64.

34. Dong Z, Pan T, Fang Y, et al. Purified CD34(+) cells versus peripheral blood mononuclear cells in the treatment of angiitis-induced no-option critical limb ischaemia: 12-month results of a prospective randomised singleblinded non-inferiority trial. EBioMedicine. 2018;35:46-57.

35. Sun L, Wu L, Qiao Z, et al. Analysis of possible factors relating to prognosis in autologous peripheral blood mononuclear cell transplantation for critical limb ischemia. Cytotherapy. 2014;16:1110-6.

36. Horie T, Onodera R, Akamastu M, et al. Long-term clinical outcomes for patients with lower limb ischemia implanted with G-CSF-mobilized autologous peripheral blood mononuclear cells. Atherosclerosis. 2010;208:461-6

Ready to submit your research? Choose BMC and benefit from:

- fast, convenient online submission

- thorough peer review by experienced researchers in your field

- rapid publication on acceptance

- support for research data, including large and complex data types

- gold Open Access which fosters wider collaboration and increased citations

- maximum visibility for your research: over $100 \mathrm{M}$ website views per year

At $\mathrm{BMC}$, research is always in progress.

Learn more biomedcentral.com/submissions 